\title{
Comparative Study between Field-in-Field and IMRT Techniques in Prostate Cancer Radiotherapy: A Treatment Planning Study
}

\author{
Tamer Dawod1, Sabbah I. Hammoury ${ }^{2 *}$ \\ ${ }^{1}$ Clinical Oncology and Nuclear Medicine Department, Faculty of Medicine, Mansoura University, Mansoura, \\ Egypt \\ ${ }^{2}$ Medical Physics Department, Alexandria Ayadi Almostakbl Oncology Center (AAAOC), Alexandria, Egypt \\ Email: tamerdawod@gmail.com, "sabbah_ibrahiem@yahoo.com
}

Received 27 October 2015; accepted 1 February 2016; published 4 February 2016

Copyright (C) 2016 by authors and Scientific Research Publishing Inc.

This work is licensed under the Creative Commons Attribution International License (CC BY). http://creativecommons.org/licenses/by/4.0/

c) (†) Open Access

\begin{abstract}
Introduction: Field-in-Field (FIF) and Intensity Modulated Radiation Therapy (IMRT) are two advanced radiation therapy planning techniques. Both of them are being used to achieve the same two related aims which are, to expose the targeted tumor to the full radiation dose and to spare the nearby normal tissues (or organs) from being exposed to high amounts of radiation more than its tolerance dose limits. FIF is a forward planning while IMRT is an inverse planning and FIF is a forward IMRT. Aim: The purpose of this study was to compare between Field-in-Field and IMRT techniques in prostate cancer radiotherapy. Method: A treatment planning system supporting both inverse and forward planning facilities is used. Ten prostate cancer patients were planned with both FIF and IMRT planning techniques. Doses received by the Planning Target Volume (PTV) and Organs at Risk (OARs) were compared in the two methods quantitatively from Dose Volume Histograms (DVHs) and qualitatively from (axial cuts). Results: The results showed that the IMRT planning technique achieved better dose coverage to the PTV than the FIF planning technique but, except RT and LT Femoral Heads, FIF achieved a better protection to the Rectum and the Bladder (OARs) than IMRT. Conclusions: The results showed that the inverse planning based IMRT technique is better and recommended in the prostate cancer radiotherapy than the FIF technique.
\end{abstract}

\section{Keywords}

Radiotherapy, IMRT, FIF, Prostate Cancer, Linear Accelerator, Treatment Planning System TPS

\footnotetext{
${ }^{*}$ Corresponding author.
}

How to cite this paper: Dawod, T. and Hammoury, S.I. (2016) Comparative Study between Field-in-Field and IMRT Techniques in Prostate Cancer Radiotherapy: A Treatment Planning Study. International Journal of Medical Physics, Clinical Engineering and Radiation Oncology, 5, 18-25. http://dx.doi.org/10.4236/ijmpcero.2016.51002 


\section{Introduction}

Three-dimensional (3D) conformal radiation therapy has been demonstrated to achieve improvement of tumor targeting and to reduce normal tissue volume exposed in several malignancies [1] [2].

To improve dose distribution, the FIF technique has been used in the treatment of certain cancers. It is a manually based forward intensity-modulated radiotherapy (forward-IMRT) plan for which the calculated dose is adjusted in certain dose distribution areas by designing multiple lower-weighted reduction fields based on the main field [3]-[5].

Intensity-Modulated Radiation Therapy (IMRT) is a radiation therapy technique in which non-uniform fluence is delivered to the patient from any given direction of the treatment beam to improve the composite dose distribution. The treatment criteria for plan optimization are determined by the planner and the optimal intensity profiles for a given set of beam directions are specified through "inverse planning" [6] [7]. The intensity files thus generated are electronically delivered to the linear accelerator (Linac), which is computer controlled, equipped with the required hardware and software to deliver the calculated intensity-modulated beams (IMBs) $[8]-[10]$.

\section{Materials and Methods}

\subsection{Acquisition and Simulation}

Ten prostate cancer patients underwent a Computed Tomography (CT) scan with $2 \mathrm{~mm}$ slice thickness. All sets of CT cuts were transferred to Focal system. Tumor volumes such as gross target volume (GTV), clinical target volume (CTV), and planning target volume (PTV) [11] as well as Organs at Risk (OARs) had been delineated by the physician on each axial slice, and then CT slices were transferred to the treatment planning system (TPS).

\subsection{Treatment Planning System}

Three-dimensional treatment planning system (TPS) Computerized Medical Systems (CMS) XiO software (release 4.64) was used to carry out dose calculation for all patients under study by super position algorithm. The TPS has both inverse and forward planning facilities. In inverse planning: after the beams are set, the inverse IMRT planning facility was used to make an automatic segmentation of all beams using the Multileaf Collimator (MLC). In forward IMRT planning (Field-in-Field (FIF)), the beam parameters were manually adjusted in a try and error process to achieve a good dose distribution to the PTV and at the same time to spare the OARs from receiving high doses.

\subsection{Beams Arrangement}

The beam arrangement was determined by the size and location of the tumor. Major seven fields with equal values of dose were used to plan each patient. In inverse planning, the beams are spread around the target with equispace and to avoid the opposing fields an odd numbers of the treatment fields were used. IMRT dose constraints for both target and OARs were entered to Xio TPS [12]-[14]. Also some conditions were given to the inverse TPS which were the minimum, goal and maximum radiation doses for the target volume, the power and weight of each structure. Several trials were taken place by Inverse TPS to achieve the IMRT dose constraints and a homogeneous dose distribution [10] [12]. Then FIF technique is used for the same selected patients where some of the manually set beams were manually segmented and one or more of sub-fields were set inside the main field(s) with small doses aiming to get rid of over doses in the main field(s) and therefore to achieve a homogeneous dose distribution.

\subsection{Evaluation of the Treatment Plans}

The treatment plans can be qualitatively and quantitatively evaluated by many tools which are already included into Xio TPS. In our study, we used two main treatment plan evaluation tools; the visual slice-by slice review of the treatment plans using isodose lines distribution as a qualitative evaluation for the treatment plans which is important to know the location of the hot and cold areas and review dose distribution to both of the clinical target and OARs and the Dose Volume Histogram (DVH) was generated to evaluate the dose to the different structures in different treatment plans and it can be used as a quantitative evaluation for the treatment plans 
[15]-[17].

\section{Results}

\subsection{FIF versus IMRT Radiotherapy Planning Techniques in Prostate Tumors}

\subsubsection{According to a Qualitative Evaluation Tool (the Axial Cuts and DRRs)}

The next Figure 1 showed colored print screens of two axial cuts and DRRs for every one of ten prostate cancer patients planned using FIF (Group (a)) and IMRT (Group (b)) radiotherapy treatment techniques. Figure 1(a) and Figure 1(b) showed the dose distribution to the treatment target (PTV), the beams arrangements, some isodose lines and how the OARs are protected from being exposed to high radiation doses in each one of the FIF and IMRT techniques. As a qualitative evaluation; from the next figure, it is noted that the IMRT achieved a better dose distribution to target of treatment (PTV) than that dose distribution achieved by the FIF. But the FIF achieved a better OARs radiation dose sparing than that achieved by the IMRT.

\subsubsection{According to a Quantitative Evaluation Tool (from the DVHs)}

1) For the PTV:

Table 1 showed the Mean and Standard Deviation $( \pm$ SD) of volume $\%$ of PTV covered with $107 \%$ of the total prescribed dose (PTV 107\%), PTV 95\%, PTV 90\%, PTV 70\%, dose \% received by $98 \%$ of PTV volume (D 98\%), D 95\%, D 50\% and D 2\% in Method 1 (FIF) and Method 2 (Inverse-IMRT) respectively. It is noticed that the Inverse-IMRT achieved higher mean values for the all mentioned PTV related results and achieved lower SD values for the same PTV related results than FIF. Figure 2 showed the Mean and SD for the Homogeneity Index (HI) of the all above PTV related results in all patients under the study in Method 1 and Method 2, and they are $(0.197 \pm 0.17,0.147 \pm 0.07)$ respectively, from which we noted that there is no significant difference between the two methods. Also Figure 3, Figure 4 showed the Mean and SD of Dose Global Max and Mean Dose to PTV in Method 1 and Method 2 which are $(98.1 \pm 1.75,100.8 \pm 0.45),(101.5 \pm 0.97,104.6 \pm 2.54)$ respectively, where the all Mean values for the two Methods are within the acceptable dose range to the PTV. It is noted that there are some big SD values in Method 1 (FIF) which are due to the resulted heterogeneous dose distribution to the PTV in FIF because of being it so closed to OARs.

2) For the OARs:

The next Table 2 showed Mean and Standard Deviation $( \pm \mathrm{SD})$ of Organs at Risk (Rectum, Bladder and Right and Left Femoral Heads) dose parameters for both Inverse-IMRT and FIF techniques respectively in all cases under the study. It is noted that FIF achieved a better sparing of both Rectum and Bladder from receiving high doses than IMRT and. But for the Right and Left Femoral Heads, the IMRT achieved better protection than FIF.

\section{Discussion}

Being of many international publications studied the use of Inverse-IMRT technique in prostate cancer radiation

Table 1. Mean and Standard Deviation $( \pm \mathrm{SD})$ of PTV dose parameters for both FIF and Inverse-IMRT.

\begin{tabular}{|c|c|c|}
\hline \multirow{2}{*}{ Measured indices from DVH } & \multicolumn{2}{|c|}{ (Mean and $\pm \mathrm{SD}$ ) respectively } \\
\hline & Method 1 (FIF) & Method 2 (Inverse-IMRT) \\
\hline V107 (\%) & $(0.037 \pm 0.089)$ & $(0.148 \pm 0.444)$ \\
\hline V95 (\%) & $(90.89 \pm 14.03)$ & $(99.56 \pm 0.592)$ \\
\hline V90 (\%) & $(96.53 \pm 7.151)$ & $(99.98 \pm 0.054)$ \\
\hline V70 (\%) & $(99.83 \pm 0.386)$ & $(100.0 \pm 0.000)$ \\
\hline D98 (Gy) & $(91.10 \pm 8.221)$ & $(97.60 \pm 1.280)$ \\
\hline D95 (Gy) & $(93.00 \pm 5.911)$ & $(98.51 \pm 0.782)$ \\
\hline D50 (Gy) & $(98.62 \pm 1.022)$ & $(100.9 \pm 0.631)$ \\
\hline D2 (Gy) & $(101.1 \pm 0.797)$ & $(103.4 \pm 1.246)$ \\
\hline
\end{tabular}



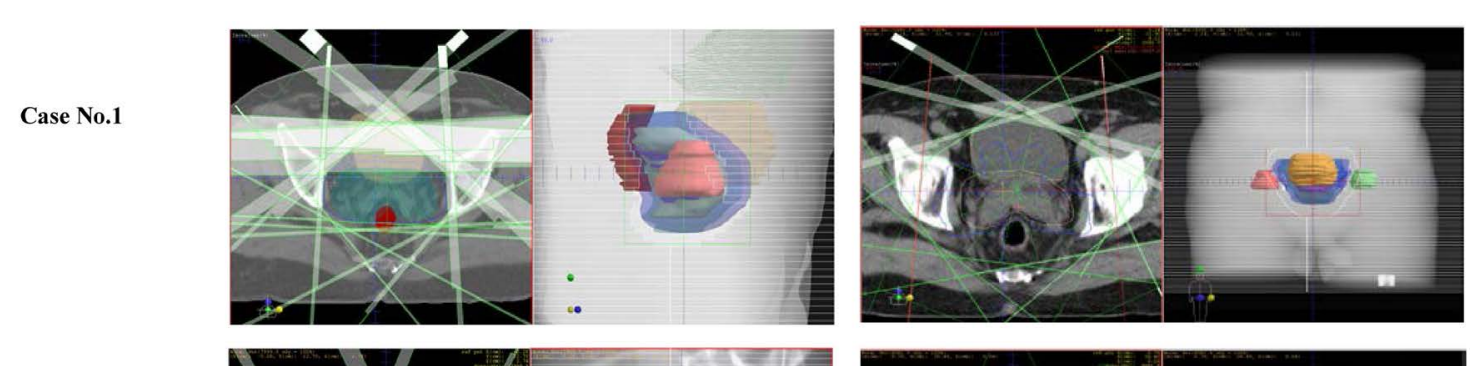

Case No. 2
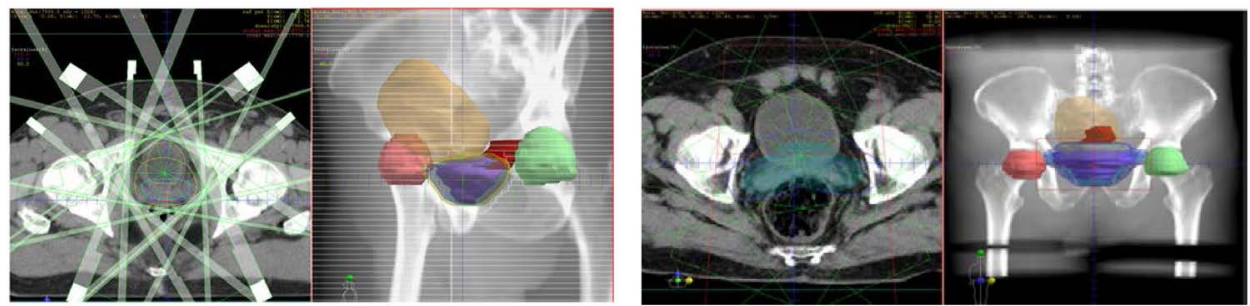

Case No. 3
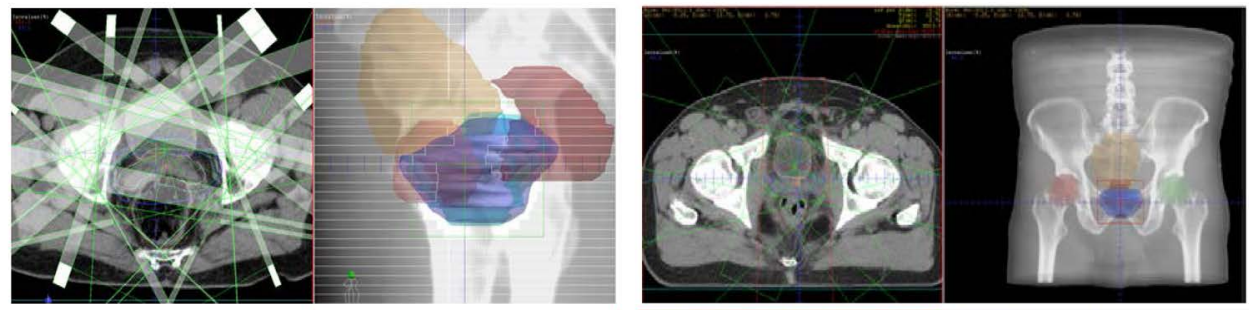

Case No. 4
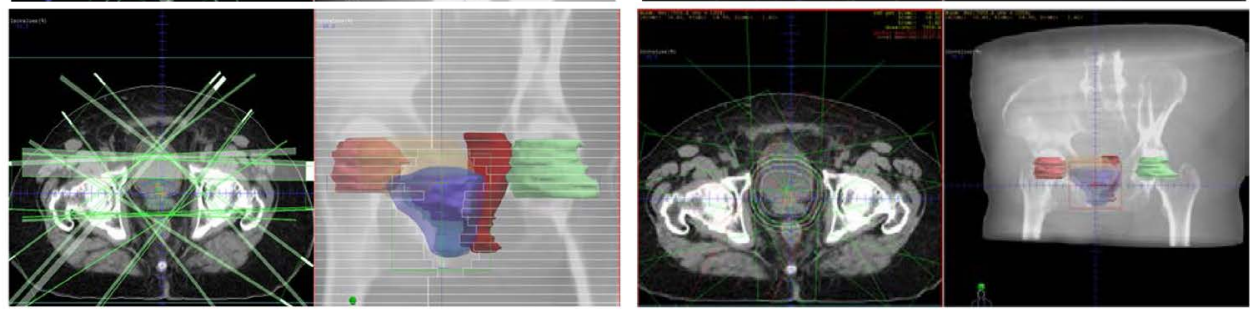

Case No. 5
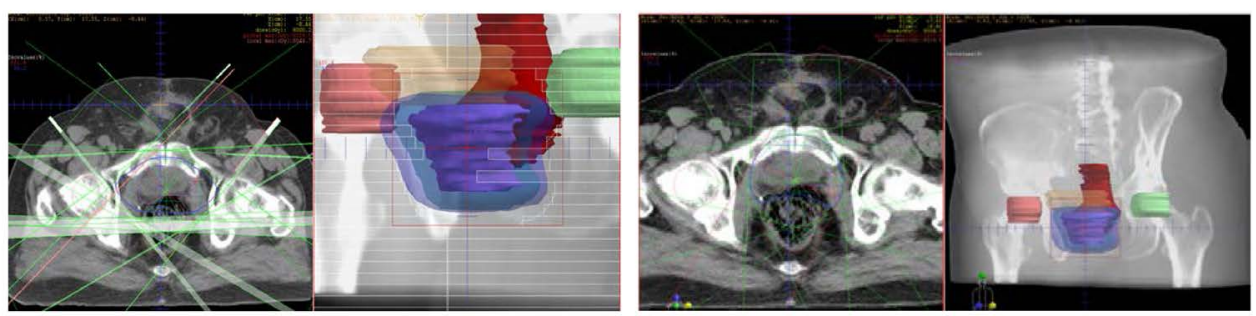

Case No. 6
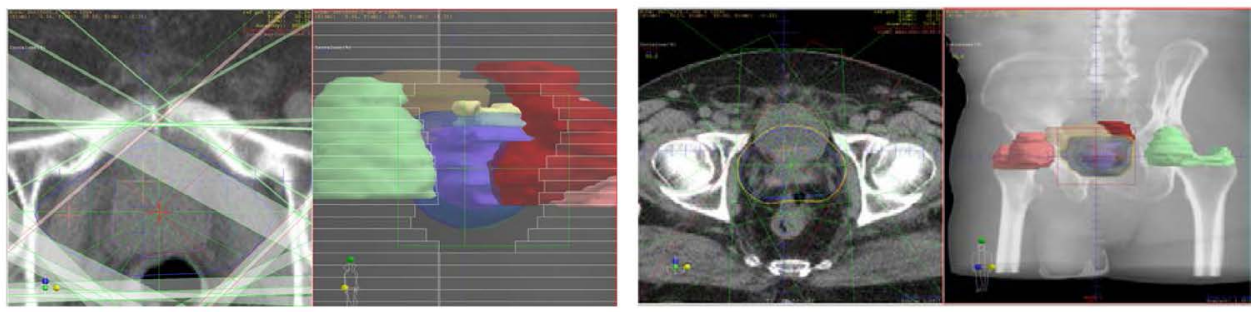

Case No. 7
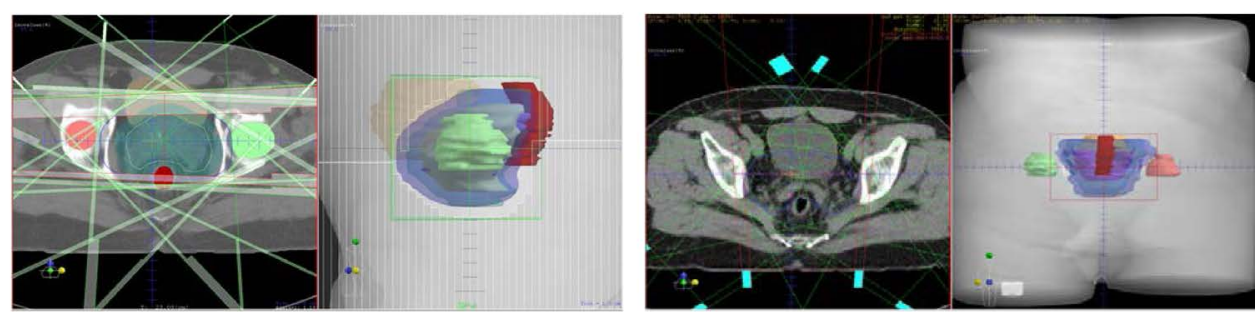
Case No. 8
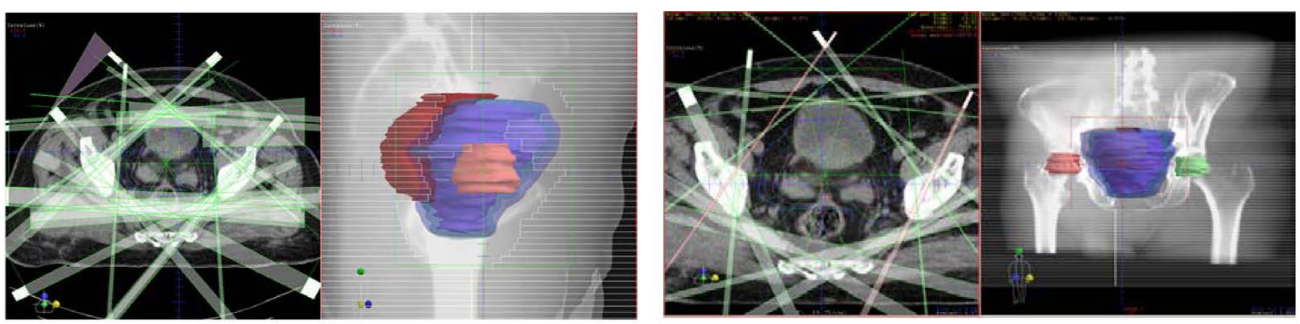

Case No. 9
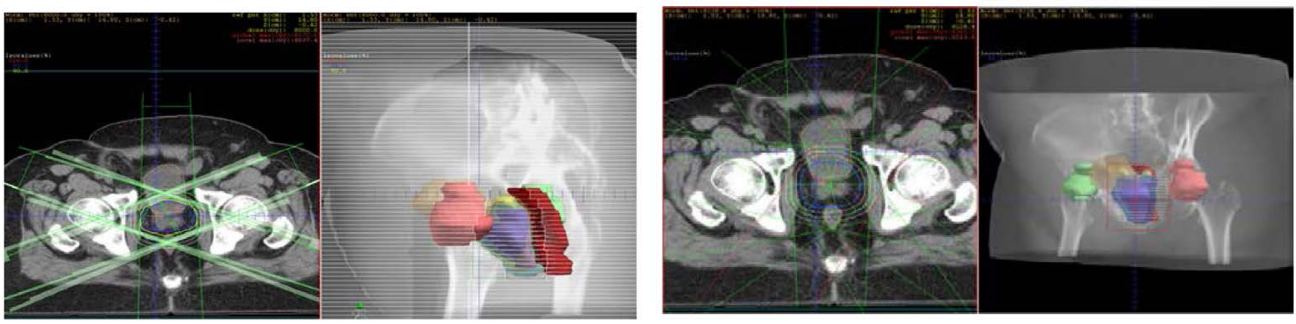

Case No. 10

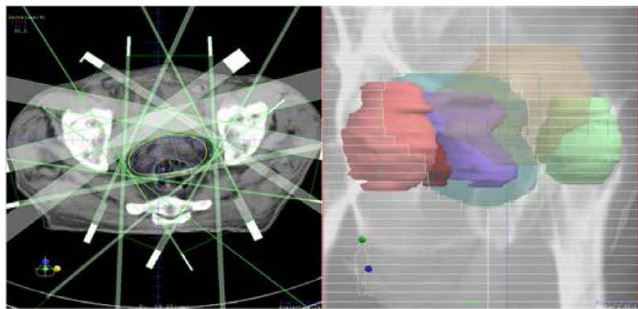

(a)

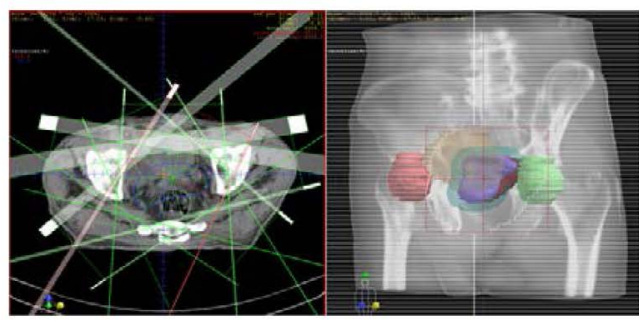

(b)

Figure 1. Group (a) and Group (b): Show two axial cuts and DRRs for every one of ten prostate cancer patients showing the beams arrangement, the dose distribution covering the PTV and protection of OARs in FIF (Group (a)) and IMRT (Group (b)) radiotherapy treatment plans.

Homogeneity Index (HI)

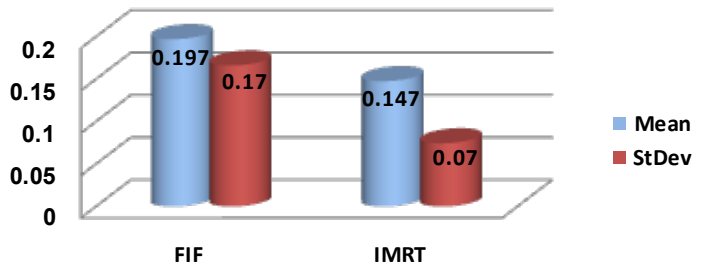

Figure 2. Mean and Standard Deviation $( \pm \mathrm{SD})$ Homogeneity Index (HI) for PTV for IMRT and FIF techniques in ten patients with Prostate tumors. Calculation formula: HI $=($ Dose Max. - Dose Min. $) /$ Dose Mean in PTV.

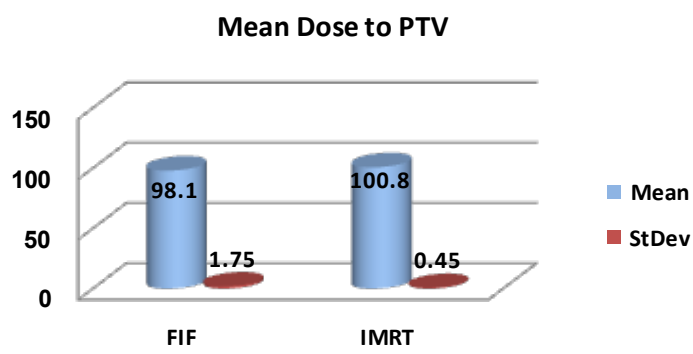

Figure 3. Mean and Standard Deviation for Mean Dose \% Received by PTV for IMRT and FIF techniques in ten patients with Prostate tumors. 


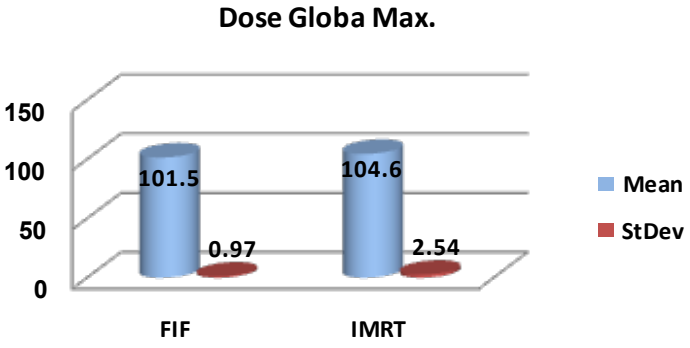

Figure 4. Mean and Standard Deviation for Dose Global Maximum \% Received by PTV for IMRT and FIF techniques in ten patients with Prostate tumors.

Table 2. Mean and Standard Deviation $( \pm \mathrm{SD})$ of Organs at Risk dose parameters for both Inverse-IMRT and FIF.

\begin{tabular}{|c|c|c|c|}
\hline \multirow{2}{*}{ OARs } & \multirow{2}{*}{ Measured indices from DVH } & \multicolumn{2}{|c|}{ (Mean and $\pm \mathrm{SD}$ ) respectively } \\
\hline & & Method 1 (FIF) & Method 2 (Inverse-IMRT) \\
\hline \multirow[t]{6}{*}{ Rectum } & D50\% & $(57.7 \pm 28.55)$ & $(63.2 \pm 26.71)$ \\
\hline & $\mathrm{D} 35 \%$ & $(69.4 \pm 26.53)$ & $(74.7 \pm 20.12)$ \\
\hline & $\mathrm{D} 25 \%$ & $(78.7 \pm 23.14)$ & $(83.6 \pm 12.97)$ \\
\hline & $\mathrm{D} 20 \%$ & $(83.5 \pm 20.72)$ & $(87.6 \pm 10.81)$ \\
\hline & D15\% & $(87.8 \pm 17.71)$ & $(91.7 \pm 8.24)$ \\
\hline & Max. Dose & $(99.3 \pm 2.12)$ & $(103.7 \pm 2.83)$ \\
\hline \multirow[t]{5}{*}{ Bladder } & D50\% & $(43.8 \pm 29.33)$ & $(52.8 \pm 32.12)$ \\
\hline & $\mathrm{D} 35 \%$ & $(57.97 \pm 29)$ & $(67.3 \pm 31.04)$ \\
\hline & $\mathrm{D} 25 \%$ & $(67.8 \pm 29.29)$ & $(77.9 \pm 30.83)$ \\
\hline & D15\% & $(78.9 \pm 26.08)$ & $(87.0 \pm 26.83)$ \\
\hline & Max. Dose & $(100.3 \pm 1.72)$ & $(103.5 \pm 0.86)$ \\
\hline \multirow[t]{2}{*}{ Right and Left Femoral Heads } & Aver. D50\% & $(43.2 \pm 14.92)$ & $(34.6 \pm 10.51)$ \\
\hline & Aver Max. Dose & $(72.6 \pm 21.14)$ & $(61.1 \pm 19.42)$ \\
\hline
\end{tabular}

therapy, the lack of the publications that discussed the use of the Field-in-Field (FIF) technique (which is a form of the Forward-IMRT) in the prostate cancer radiotherapy was the main reason of choosing the point of this study. Also it is worth to be mentioned that we noted that many centers like our centers at which we are work, still use the FIF more than they use the IMRT in prostate cancer radiotherapy.

\section{Conclusion}

According to the all previous results, we conclude that the IMRT planning technique achieved better dose coverage to the PTV than the FIF planning technique but except RT and LT Femoral Heads, FIF achieved a better protection to the Rectum and the Bladder (OARs) than IMRT. So we conclude and recommend that the IMRT technique is better in the prostate cancer radiotherapy than the FIF technique [18]-[22].

\section{Limitations}

There are no limitations regarding to the application of this study for treating the prostate cancer patients as long as the Inverse-IMRT planning was available. 


\section{Acknowledgements}

The authors thank Prof. Dr. Assem Rostom, consultant clinical oncologist, the general manager of Alexandria Ayadi Almostakbl Oncology Centre (AAAOC) and Dr. Mostafa Ali Elnaggar, the medical manager of Alexandria Ayadi Almostakbl Oncology Centre (AAAOC), for their continuous and valuable support and encouragement.

\section{References}

[1] Morris, D.E., Emami, B., Mauch, P.M., Konski, A.A., Tao, M.L., et al. (2005) Evidence-Based Review of Three-Dimensional Conformal Radiotherapy for Localized Prostate Cancer: An ASTRO Outcomes Initiative. International Journal of Radiation Oncology Biology Physics, 62, 3-19. http://dx.doi.org/10.1016/j.ijrobp.2004.07.666

[2] Nilsson, S., Norlén, B.J. and Widmark, A. (2004) A Systematic Overview of Radiation Therapy Effects in Prostate Cancer. Acta Oncologica, 43, 316-381. http://dx.doi.org/10.1080/02841860410030661

[3] Prabhakar, R., Haresh, K.P., Kumar, M., Sharma, D.N., Julka, P.K. and Rath, G.K. (2009) Field-in-Field Technique for Upper Abdominal Malignancies in Clinical Radiotherapy. Japanese Journal of Radiology, 28, 20-23. http://dx.doi.org/10.4103/0973-1482.48765

[4] Alison, L.S., Tomas, K., Alan, H., Colin, H., Jim, C., Kelly, S., et al. (2011) Does Inverse-Planned Intensity-Modulated Radiation Therapy Have a Role in the Treatment of Patients with Left-Sided Breast Cancer? Journal of Medical Imaging and Radiation Oncology, 55, 311-319. http://dx.doi.org/10.1111/j.1754-9485.2011.02273.x

[5] Cox, J.D. and Ang, K.K. (2010) Radiation Oncology: Rationale, Technique, Results. 9th Edition.

[6] Meerleer, G.O., Vakaet, L.A., Bate, M.T., Wagter, C., Naeyer, B. and Neve, W.J. (1999) The Single Isocenter Treatment of Head and Neck Cancer: Time Gain Using MLC and Automatic Set-Up. Cancer Radiothérapie, 3, $235-241$. http://dx.doi.org/10.1016/S1278-3218(99)80057-2

[7] Prado, K.L., Starkschall, G. and Mohan, R. (2007) Three-Dimensional Conformal Radiation Therapy. In: Khan, F.M., Ed., Treatment Planning in Radiation Oncology, 2nd Edition, Chapter 10, 116-141.

[8] Boyer, A., Xing, L., Luxton, G. and Ma, C.-M. (2000) Dynamic IMRT Is Sufficient for All Clinical Purposes. Proceedings of the 13th International Conference on the Use of Computers in Radiation Therapy, Heidelberg, May 2000, 7-9.

[9] Webb, S. (2001) Intensity Modulated Radiation Therapy. Institute of Physics (IOP) Publishing, Bristol.

[10] Alanc, H.A., Adelineg, M., Alisca, P., Thomas, J., Ichler, E., David, C., et al. (2009) American Society for Therapeutic Radiology and Oncology (ASTRO) and American College of Radiology (ACR) Practice Guidelines for IntensityModulated Radiation Therapy (IMRT). International Journal of Radiation Oncology Biology Physics, 73, 9-14. http://dx.doi.org/10.1016/j.ijrobp.2008.04.049

[11] ICRU, Bethesda MD. (1999) International Commission on Radiological Units, Prescribing, Recording and Reporting Photon Beam Therapy. Supplement to ICRU 50, ICRU Report 62.

[12] Guckenberger, M., Pohl, F., Baier, K., Meyer, J., Vordermark, D. and Flentje, M. (2006) Adverse Effect of a Distended Rectum in Intensity-Modulated Radiotherapy (IMRT) Treatment Planning of Prostate Cancer. Radiotherapy and Oncology, 79, 59-64. http://dx.doi.org/10.1016/j.radonc.2006.03.004

[13] Sarah, R., Wim, D., Karin, H., et al. (2006) Definition and Delineation of the Clinical Target Volume for Rectal Cancer. International Journal of Radiation Oncology Biology Physics, 65, 1129-1142. http://dx.doi.org/10.1016/j.ijrobp.2006.02.050

[14] Chui, C.S., Chan, M.F., Yorke, E., Spirou, S. and Ling, C.C. (2001) Delivery of Intensity-Modulated Radiation Therapy with a Conventional Multileaf Collimator: Comparison of Dynamic and Segmental Methods. Medical Physics, 28, 2441-2449. http://dx.doi.org/10.1118/1.1418018

[15] Jin, G.-H., Chen, L.-X., Deng, X.-W., Liu, X.-W., Huang, Y. and Huang, X.-B. (2013) A Comparative Dosimetric Study for Treating Left-Sided Breast Cancer for Small Breast Size Using Five Different Radiotherapy Techniques: Conventional Tangential Field, Filed-in-Filed, Tangential-IMRT, Multi-Beam IMRT and VMAT. Radiation Oncology, 8, 89. http://dx.doi.org/10.1186/1748-717X-8-89

[16] Fallon Community Health Plan. (2009) Conformal and Intensity Modulated Radiation Therapy (IMRT) of the Breast. Policy No. 200909-0002, 1-9.

[17] Alison, L.S., Tomas, K., Alan, H., et al. (2011) Does Inverse-Planned Intensity-Modulated Radiation Therapy Have a Role in the Treatment of Patients with Left-Sided Breast Cancer? Journal of Medical Imaging and Radiation Oncology, 55, 311-319. http://dx.doi.org/10.1111/j.1754-9485.2011.02273.x

[18] Ercan, T., et al. (2010) Dosimetric Comparison of Field in Field Intensity-Modulated Radiotherapy Technique with 
Conformal Radiotherapy Techniques in Breast Cancer. Japanese Journal of Radiology, 28, 283-289. http://dx.doi.org/10.1007/s11604-010-0423-3

[19] Intensity-Modulated Radiation Therapy: Medical Policy (2014) Proprietary Information of United Healthcare. Policy No. 2014T0407M.

[20] Bucci, M.K., Alison, B. and Mack, R. (2005) Advances in Radiation Therapy: Conventional to 3D, to IMRT, to 4D, and Beyond. CA: A Cancer Journal for Clinicians, 55, 117-134. http://dx.doi.org/10.3322/canjclin.55.2.117

[21] Khan, F.M. (2010) Physics of Radiation Therapy. 4th Edition, Lippincott Williams \& Wilkins, Philadelphia, 431-432.

[22] Olivier, R., Pauline, R.M., David, A., Norbert, A., Jean-Bernard, D. and Pascal, F. (2013) Simultaneous Integrated Boost Plan Comparison of Volumetric-Modulated Arc Therapy and Sliding Window Intensity-Modulated Radiotherapy for Whole Pelvis Irradiation of Locally Advanced Prostate Cancer. Journal of Applied Clinical Medical Physics, 14, 26-32. http://www.jacmp.org/index.php/jacmp/article/download/4094/2967

\section{Abbreviations and Acronyms}

IMRT: Intensity-Modulated Radiation Therapy

AAAOC: Alexandria Ayadi Almostakbl Oncology Center

FIF: $\quad$ Field-in-Field

PTV: $\quad$ Planning Target Volume

OARs: $\quad$ Organs at Risk

DVHs: Dose Volume Histograms

3D: $\quad$ Three-dimensional

Linac: Linear accelerator

IBMs: Intensity-Modulated Beams

CT: $\quad$ Computed Tomography

GTV: $\quad$ Gross Target Volume

CTV: $\quad$ Clinical Target Volume

TPS: $\quad$ Treatment Planning System

CMS: $\quad$ Computerized Medical System

XiO: $\quad$ Name of three dimensions treatment planning system

SD: $\quad$ Standard Deviation

HI: $\quad$ Homogeneity Index 